\title{
Utilização de Sistemas, Técnicas e Ferramentas de Gestão da Qualidade em Organizações de Saúde Acreditadas no Brasil
}

\author{
Use of Systems, Technical and Tools of Quality Management on Accredited Healthcare \\ Organizations in Brazil
}

\section{Uso de sistemas, técnicas y de gestión de la calidad Herramientas en Organizaciones de Salud acreditados en Brasil}

\begin{tabular}{|l|l|}
\hline Antônio Carlos Rodrigues & Submetido em: 26/06/2015 \\
Professor da Universidade Federal de Juiz de Fora - UFJF & Artigo aceito em:20/10/2015 \\
$\begin{array}{l}\text { Doutorando em Administração da Universidade Federal de Minas Gerais - UFMG } \\
\text { antonio.rodrigues@ facc.ufff.br }\end{array}$ & \\
& DOI 10.21450/rahis.v13i1.2947 \\
Mariana Luísa da Costa Lage & \\
Professora da Universidade Federal de Juiz de Fora - UFJF & \\
$\begin{array}{l}\text { Doutoranda em Administração da Universidade Federal do Espírito Santo - UFES } \\
\text { mariana.lage@ ufjf.edu.br }\end{array}$ & \\
\hline
\end{tabular}

\section{Resumo}

As técnicas e ferramentas da qualidade exercem papel crucial no sucesso da aplicação prática dos princípios e definições na Gestão da Qualidade em qualquer organização. Nesse contexto, o objetivo deste estudo é apresentar o resultado de um survey em que se verificam as principais características do processo de Acreditação, seus benefícios, suas dificuldades e quais técnicas e ferramentas da qualidade utilizadas por 84 organizações brasileira certificadas pela Organização Nacional de Acreditação (ONA). A pesquisa relevou que a Acreditação proporciona benefícios significativos às organizações: melhoria no controle dos processos da organização, aumento na segurança do paciente e melhoria no gerenciamento de recursos. Ela também ratificou a importância do uso das técnicas e ferramentas da qualidade como forma de adequação aos requisitos da Acreditação. As ferramentas mais utilizadas foram procedimento operacional padrão, fluxograma e $5 \mathrm{~W} 2 \mathrm{H}$ dentre as técnicas destacam-se ciclo PDCA, FMEA e CEP. As dificuldades encontradas foram: ausência de indicadores, burocracia excessiva e resistência dos funcionários. Apenas a ausência de indicadores, dentre todas apresentadas, mereceu destaque nesta pesquisa.

Palavras-chaves: gestão da qualidade, acreditação, técnicas e ferramentas da qualidade

\begin{abstract}
The quality techniques and tools have crucial part in the success of the practical application of principles and definitions on the Quality Management in any organization. In this context, the aim of this study is to present the result of a survey in which there are main features of Accreditation process, their benefits, difficulties and what quality techniques and tools were used in 84 brazilian organizations certified by Organização Nacional de Acreditação (ONA). This research showed that Accreditation provides significant benefits to organizations: better control of the organization's processes, increased patient safety and improved resource management. It also ratified the importance of using techniques and quality tools as a form to adapt to the Accreditation requirements. The most used tools were standard operating procedure, flowchart and 5W2H from the technical we highlight PDCA, FMEA and CEP. The difficulties were: absence of indicators, excessive bureaucracy and resistance from employees. Only the absence of indicators, among all presented, was highlighted in this research.
\end{abstract}

Key words: quality management, accreditation, quality technical and tools

\section{Resumen}

Las técnicas y herramientas de calidad desempeñan un papel crucial en el éxito de la aplicación práctica de los principios y definiciones sobre la Gestión de la Calidad en cualquier organización. En este contexto, el objetivo de este estudio es presentar los resultados de una survey donde se encuentran las principales características del proceso de Acreditación, sus beneficios, sus dificultades y cuales son las técnicas y herramientas de calidad utilizadas por 84 organizaciones brasileñas certificadas por la Organização Nacional de Acreditação (ONA). La investigación cedió que la Acreditación proporciona importantes beneficios a las organizaciones: mejor control de los procesos de la organización, aumento de la seguridad del paciente y mejor gestión de los recursos. Además ratificó la importancia del uso de las técnicas y herramientas de calidad a fin de adaptarse a los requisitos de Acreditación. Las herramientas más utilizadas eran un procedimiento operativo estándar, diagrama de flujo y 5W2H, por la técnica se destacan PDCA, FMEA y CEP. Las dificultades fueron: la falta de indicadores, el exceso 
de burocracia y la resistencia por parte de los empleados. Sólo la falta de indicadores, entre todos los presentados, se destacó en esta investigación.

Palabras-Clave: Gestión de la calidad, acreditación, técnico y herramientas de calidad

\section{Introdução}

As necessidades de cuidados, investimentos e gastos com saúde tendem a aumentar gradativamente em função de maior conscientização, por parte dos indivíduos, de que devem se cuidar melhor; de maior disponibilidade de recursos tecnológicos; de aumento do poder de renda da população e de elevação da expectativa de vida (PEDROSO; MALIK, 2011).

A assistência médica se desenvolve em ambientes dinâmicos e especializados com interações entre paciente, equipe médica, infraestrutura, equipamentos, processos, normas e procedimentos organizacionais. Geralmente, tratam-se de ações que demandam conhecimento científico especializado ou habilidade técnica validada, precisam ser implantadas rapidamente, em circunstâncias não planejadas ou não controladas por aqueles que necessitam de cuidados médicos (PEDREIRA, 2006).

Devido a grande complexidade dos serviços de saúde e o grande número de intervenções que um paciente está sujeito, a incidência de erros é algo inerente ao processo, com o agravante de que erros neste setor podem gerar danos irreversíveis (KOHN; CORRIGAN; DONALDSON, 2000, RODRIGUES et al., 2011).

Nesse sentido, a temática da gestão da qualidade ocupa um papel importante nas discussões sobre os rumos da saúde (MALIK; SCHIESARI, 1998; RODRIGUES et al., 2011). É por meio dela que as organizações de saúde são dotadas de mecanismos para planejar, controlar e melhorar seus processos continuamente (COUTO; PEDROSA, 2003).

Um dos principais meios para a introdução e sistematização da filosofia e dos procedimentos da qualidade nas organizações são os Sistemas de Gestão da Qualidade (SGQs) (CARVALHO; PALADINI, 2012). Os SGQs enfatizam o desenvolvimento, implantação, padronização, manutenção e melhoria da qualidade de processos, produtos e serviços (CARPINETTI, 2012; CARVALHO; PALADINI, 2012). No ambiente de saúde a Acreditação é um dos SGQs mais utilizados pelas organizações de saúde, como: hospitais, farmácias, laboratórios, serviços de imagem, serviços de hemoterapia, serviços de nefrologia, serviços ambulatoriais e serviços de atendimento domiciliar (ONA, 2010). A Acreditação ganhou importância nos Estados Unidos na década de 50, com a atuação da Joint Commission on Accreditation of Health Care Organization (JCAHO), e no Brasil, na década de 90, com a Organização Nacional de Acreditação (ONA). As organizações brasileiras desenvolvem já há alguns anos, instrumentos oficiais de avaliação de desempenho das organizações de saúde tendo por base a aplicação de conceitos e técnicas da qualidade total (QUINTO NETO; GASTAL, 2000).

Sobretudo, um dos elementos que permitem operacionalização dos preceitos destes modelos gerenciais são as técnicas e as ferramentas (T\&Fs) da qualidade (BAMFORD; GREATBANKS, 2005). São elas que permitem o desenvolvimento, mensuração, análise e melhoria da qualidade nas organizações. As técnicas e ferramentas da qualidade permitem a identificação e solução dos principais problemas organizacionais e, por este motivo, são importantes instrumentos de diferenciação organizacionais (HELLSTEN; KLEFSJÖ, 2000).

Contudo, a utilização tanto dos SGQs e T\&Fs enfrenta uma série de dificuldades em face das características do ambiente organizacional: deficiências em relação à qualificação de mão de obra, baixo fôlego financeiro de médias e pequenas empresas e ausência de programas públicos e/ou coletivos de fomento da competitividade empresarial (BAMFORD; GREATBANKS, 2005).

Pelo exposto, a questão de pesquisa que norteará o desenvolvimento deste trabalho é a seguinte: a acreditação gera na prática os benefícios elencados na literatura e fomenta a 
utilização de técnicas e ferramentas nas organizações de saúde brasileiras? Nesse sentido, o objetivo do trabalho será apresentar o resultado de um survey em que foram verificadas e analisadas as principais características do processo de acreditação, seus benefícios, suas dificuldades de implantação e quais $T \& F s$ da qualidade utilizadas em organizações brasileiras.

$\mathrm{O}$ restante do artigo está dividido em cinco seções. Na seção 2, é apresentado o referencial teórico sobre: avaliação e acreditação em organizações de saúde, Organização Nacional de Acreditação (ONA) e técnicas e ferramentas da qualidade. Na seção 3, é exposta a metodologia utilizada no trabalho. Na seção 4, os dados são analisados e os resultados, discutidos. Finalizando o artigo, a seção 5 apresenta as conclusões do trabalho.

\section{Referencial Teórico}

1.1.Avaliação e acreditação em organizações de saúde

Considerando que as atividades desenvolvidas pelas organizações de saúde carregam consigo um potencial de dano implícito, os profissionais envolvidos na assistência estão obrigados a uma busca constante de modelos de melhoria e de garantia da qualidade na gestão da atenção à saúde oferecida à população, seja nos procedimentos, seja nos processos internos, seja nas escolhas de materiais, medicamentos e demais recursos tecnológicos (PEDREIRA, 2006; PEDROSA; COUTO, 2007; RODRIGUES et al., 2011). Esta complexidade, difícil de observar em outros setores da economia, gera dificuldades na avaliação de serviços e processos de produção cuja padronização é sempre um desafio.

As características tradicionais dos serviços (intangibilidade, inseparabilidade, heterogeneidade e perecibilidade) dificultam a escolha e a avaliação do que se deseja em termos da prestação destes. Não obstante, os serviços de saúde são ainda mais difíceis de escolher e avaliar pela importante falta de conhecimento a respeito do serviço apresentada pela parte "contratante" (CORREA; CANON, 2009; FITZSIMMONS; FITZSIMMONS, 2014; BERRY; PARASURAMAN; ZEITHAML; 2014).

O processo avaliativo dos serviços pode ser caracterizado de duas maneiras: a avaliação formativa, que é de caráter contínuo e interno; e a somativa ou classificatória, que depende das informações internas e do concurso de profissionais externos para a realização dos procedimentos avaliativos propriamente ditos (RODRIGUES et al., 2011).

Nas organizações de saúde, com o processo ocorrendo continuamente por meio da interação entre os grupos, a construção coletiva do saber gera forte potencialidade para a gestão do conhecimento e obtenção de vantagens competitivas.

Por definição, identifica-se que as possibilidades de avaliação passam por modelos de construção que podem ser internos ou externos. Cada qual tem as suas particularidades e vantagens. O modelo de avaliação interna, coincidente com a avaliação formativa, pode ser utilizado mesmo em ambientes nos quais não se queira falar abertamente sobre programas de qualidade, avaliação e pesquisas afins. Existem organizações que passaram por processos traumáticos de avaliação e de programas de qualidade com reflexos sobre o corpo vivo da organização. Os modelos de avaliação interna pressupõem uma capacidade de adaptação à realidade local que permite "atingir os padrões externos" sem a pressão de ter que se submeter aos programas externos e aos seus consultores (RODRIGUES et al., 2011).

O alcance dos padrões externos, entretanto, denota que é realizado um tipo de benchmarking sobre os modelos externos de avaliação e que eles têm uma vantagem marcante sobre os modelos internos: a partir do concurso de especialistas, é criada uma metodologia, com bases teóricas consistentes e que é desenvolvida de modo a servir para todas as organizações do setor, utilizando-se de critérios e definições que sejam externas à organização e comuns ao setor (RODRIGUES et al., 2011). 
Nos serviços de saúde, o modelo externo, com especialistas definindo o que é qualidade, fornecendo padrões e critérios de reconhecimento capazes de garantir a segurança do usuário do sistema de saúde, com olhar crítico e sistemas de manutenção e renovação das avaliações, e por fim, terminou por estabelecer-se como referência e é chamado de acreditação.

Acreditar significa ter confiança, dar ou estabelecer crédito e embute em si tranquilidade, segurança e satisfação (RODRIGUES et al., 2011).

A acreditação constitui uma metodologia pautada na racionalização, organização e de educação permanente do pessoal e de líderes das organizações prestadoras de serviços de saúde. É fundamentado na avaliação dos padrões de referências desejáveis, construídos por profissionais da área e previamente divulgados, e nos indicadores ou instrumentos que o avaliador emprega para constatar os padrões que estão sendo observados (ONA, 2010).

Por outro lado, certificar significa, por sua vez, atestar ou afirmar que determinada ocorrência se dá em conformidade com o esperado (RODRIGUES et al., 2011).

Segundo Rooney e Van Ostenberg (1999), metodologicamente, a acreditação, apesar de também ser uma forma de certificação, difere um pouco da certificação tradicionalmente aceita em programas de qualidade, uma vez que busca apenas definir padrões de conformidade com determinada norma, determinação ou proposição, que são aferidos para a obtenção do certificado.

Enquanto isso, a acreditação de uma organização, para que possa conferir a credibilidade esperada como processo, tem a necessidade da intervenção de avaliadores externos, com atuação direcionada para a observação das minúcias da organização e especial referência à estrutura, aos processos e resultados.

Ao contrário dos processos tradicionais de avaliação, a acreditação possui uma forte abordagem educativa, baseada na reflexão da prática profissional, que leva a elaboração de padrões de excelência de desempenho. Por ser um processo, sobretudo reflexivo, sempre revela novas formas de visualizar e agir sobre os problemas da instituição (ROONEY; OSTENBER, 1999; RODRIGUES et al., 2011).

Além disso, cabe ressaltar que, diferentemente de outras formas de certificação, na acreditação a definição dos padrões de qualidade é externa à organização e comum ao setor, sempre definida por especialistas, e capaz de afirmar e confirmar o que é e o que não é qualidade (RODRIGUES et al., 2011; ONA, 2010).

Por fim, a acreditação de serviços de saúde apresenta-se para os gestores de sistemas de saúde com duas importantes dimensões que devem ser exploradas: dimensão educacional, que consiste em fazer com que as organizações introduzam a questão da qualidade em suas agendas, apropriando a cultura da qualidade em serviços de saúde; dimensão certificadora, que permite avaliar e atestar a qualidade existente nas organizações de saúde a partir dos padrões externos definidos pelos especialistas (RODRIGUES et al., 2011).

\subsection{Organização Nacional de Acreditação}

A Organização Nacional de Acreditação (ONA) é uma organização privada, sem fins lucrativos e de interesse coletivo, cuja é a promoção de um processo de acreditação, visando aprimorar a qualidade da assistência na área da saúde no Brasil (ANTUNES, 2002). Ela é responsável pelo estabelecimento de padrões e pelo monitoramento do processo de acreditação realizado pelas instituições acreditadoras.

O Programa Brasileiro de Acreditação Hospitalar (PBAH), por sua vez, visa o amplo entendimento de um processo permanente de melhoria da qualidade assistencial, mediante a acreditação periódica da rede hospitalar pública e privada. Para tanto, instituiu no âmbito hospitalar mecanismos para autoavaliação e aprimoramento contínuo da qualidade da atenção médico-hospitalar (ONA, 2010). 
Na lógica do Processo de Acreditação (PAc) não se avalia um serviço ou departamento isoladamente. Entende-se que as estruturas e processos do hospital são de tal ordem interligada que o funcionamento de um componente interfere em todo o conjunto e no resultado final (PAGANINI; NOVAES, 1992).

As Organizações Prestadoras de Serviços de Saúde (OPSS) são definidas como entidades jurídicas e legalmente constituídas, nas quais se prestam serviços de assistência médica do tipo hospitalar, hemoterápico, laboratorial e patologia clínica, ambulatorial e pronto atendimento, diagnóstico e terapia, atenção primária à saúde e assistência domiciliar, de caráter estatal ou privado, com ou sem fins lucrativos, sob a responsabilidade de uma diretoria.

As Instituições Acreditadoras (IAC) são entidades de direito privado, credenciadas pela ONA para desenvolverem o Processo de Avaliação (PAv) das OPSS e tem como atribuições e competências, avaliar a qualidade dos serviços de saúde, certificar as OPSS acreditadas e capacitar avaliadores para o PAv.

O avaliador é o profissional qualificado para efetuar as avaliações do PAc, capacitado conforme as normas definidas pela ONA. O avaliador líder é o responsável perante a IAC por todas as fases do PAv. A equipe de avaliadores deve ser composta de no mínimo, três membros, sendo: um médico, um enfermeiro e um administrador(a).

O PAc se estrutura por níveis 1, 2 e 3, com seus princípios norteadores e por padrões, sendo que cada padrão representa uma definição e uma lista de itens de verificação que permitem a identificação precisa do que avaliar e a concordância com o padrão estabelecido (ver Quadro 1).

Quadro 1 - Princípios de cada nível de certificação

\begin{tabular}{|c|c|c|}
\hline NÍVEL & PRINCÍPIO ESPECÍFICO & DESCRIÇÃO \\
\hline 1 & SEGURANÇA & $\begin{array}{l}\text { As exigências deste nível contemplam o atendimento aos requisitos básicos da } \\
\text { qualidade na assistência prestada ao cliente, nas especialidades e nos serviços } \\
\text { da organização de saúde a ser avaliada, com recursos humanos compatíveis } \\
\text { com a complexidade, qualificação adequada (habilitação) dos profissionais e } \\
\text { responsáveis técnicos com habilitação correspondente para as áreas de atuação } \\
\text { institucional. }\end{array}$ \\
\hline 2 & $\begin{array}{l}\text { SEGURANÇA e } \\
\text { ORGANIZAÇÃO }\end{array}$ & $\begin{array}{l}\text { As exigências deste nível contemplam evidências de adoção do planejamento } \\
\text { na organização da assistência, referentes à documentação, corpo funcional } \\
\text { (força de trabalho), treinamento, controle, estatísticas básicas para a tomada de } \\
\text { decisão clínica e gerencial, e práticas de auditoria interna. }\end{array}$ \\
\hline 3 & $\begin{array}{l}\text { SEGURANÇA, } \\
\text { ORGANIZAÇÃO e PRÁTICAS } \\
\text { DE GESTÃO DE } \\
\text { QUALIDADE }\end{array}$ & $\begin{array}{l}\text { As exigências deste nível contêm evidências de políticas institucionais de } \\
\text { melhoria contínua em termos de: estrutura, novas tecnologias, atualização } \\
\text { técnico-profissional, ações assistenciais e procedimentos médicos-sanitários. } \\
\text { Evidências objetivas de utilização da tecnologia da informação, disseminação } \\
\text { global e sistêmica de rotinas padronizadas e avaliadas com foco na busca da } \\
\text { excelência. }\end{array}$ \\
\hline
\end{tabular}

Fonte: ONA (2010)

Assim, uma característica marcante da metodologia da ONA é a avaliação por níveis crescentes de complexidade. Assim, no nível 1 o atributo a ser alcançado é a segurança, assemelhando-se à lógica da Agência Nacional de Vigilância Sanitária (Anvisa). No nível 2, a organização é o atributo a ser alcançado, assemelhando-se aos critérios da International Standardization Organization (ISO). E, no nível 3, o atributo a ser alcançado é a excelência, assemelhando-se ao Prêmio Nacional da Qualidade (PNQ) (RODRIGUES et al., 2011).

Nessa lógica de acreditação, cada um dos setores é avaliado individualmente, porém só se acredita uma organização de prestadora de serviço de saúde quando todos os serviços e/ou unidades atingirem os padrões definidos. Assim, a organização será acreditada de acordo com o nível mínimo de referência que atingir em cada seção. Assim, se algumas estiverem no nível 2 e as demais no 3, a instituição será acreditada no nível 2.

1.3.Técnicas e ferramentas da qualidade 
A Gestão da Qualidade tem como objetivos a padronização de processos, por meio de planejamento, controle e aprimoramento, e a garantia da qualidade de produtos e serviços. As empresas necessitam adotar um sistema que priorize a qualidade em suas decisões para que seja possível alcançar e manter a qualidade de seus processos, produtos e serviços (CARPINETTI, 2012).

A literatura demonstra que os sistemas de gestão da qualidade beneficiam significativamente às empresas (CARPINETTI; GEROLAMO; MIGUEL, 2011; CARPINETTI, 2012). No entanto, existem grandes dificuldades na adoção desses sistemas. Um dos principais benefícios, diz respeito à melhoria nos processos, produtos e serviços, porém se destaca ainda o aumento da satisfação dos clientes, melhoria da imagem da empresa, abertura de novos mercados e maior vantagem competitiva diante da concorrência. No que tange às dificuldades, observam-se as seguintes: resistência à mudança, dificuldade no entendimento e aceitação de determinados preceitos da qualidade, falta de comprometimento da média gerência e baixo envolvimento da alta direção, incluindo aporte pequeno de capital para as atividades associadas à qualidade (CARPINETTI; GEROLAMO; MIGUEL, 2011; CARPINETTI, 2012; CARVALHO; PALADINI, 2012).

Não obstante, as técnicas e ferramentas (T\&Fs) da qualidade exercem um papel essencial no êxito da aplicação prática dos princípios e definições que caracterizam esta área: elas asseguram a plena viabilização da estrutura conceitual e das diretrizes básicas da Gestão da Qualidade. Por isso, se a consistência teórica e a ampla aceitação tem sido essenciais no êxito da Gestão da Qualidade, também se pode afirmar que as ferramentas a ela associadas constituíram as formas práticas que viabilizaram o emprego desses pressupostos (CARVALHO; PALADINI, 2012).

As T\&Fs da qualidade são maneiras, competências, meios ou mecanismos práticos utilizados na realização de tarefas específicas (McQUATER et al., 1995). McQuater et al. (1996) descreve as técnicas e ferramentas da qualidade como um meio de conduta, habilidades e instrumentos práticos aplicáveis a tarefas específicas, incluindo a facilitação de mudanças ou melhorias.

Utilizadas para desenvolver, implantar, monitorar e melhorar os preceitos da qualidade nas organizações, as técnicas e ferramentas da qualidade representam importantes e necessários instrumentos para que os sistemas de gestão qualidade obtenham máxima eficiência e eficácia (BAMFORD; GREATBANKS, 2005). Avaliar a satisfação do cliente, visando à melhoria da qualidade nos serviços e produtos, é uma das importantes funções competitivas das técnicas e ferramentas da qualidade. Essa prática promove a confiabilidade e produz consideráveis vantagens à empresa em relação aos seus concorrentes (CARVALHO; PALADINI, 2012).

As T\&Fs da qualidade têm sido desenvolvidas e aprimoradas para sustentar a aplicação e utilização da gestão da qualidade nas empresas, dessa forma, considera-se que elas são imprescindíveis para o SGQ obter o sucesso largamente anunciado na literatura científica (HELLSTEN; KLEFSJÖ, 2000).

Uma técnica pode ser vista como um número de atividades executadas em certa ordem na organização para se alcançarem os princípios dos SGQs. Segundo McQuater et al. (1995) e Hellsten e Klefsjö (2000), uma técnica possui uma aplicação mais ampla que uma ferramenta. Isto geralmente demanda uma maior reflexão, habilidade e treinamento. Elas consistem em um número de atividades realizadas em certa ordem. De forma resumida, as técnicas podem ser pensadas como uma coleção de ferramentas. Exemplos de técnicas são: Desdobramento da função da Qualidade (QFD), Desenho de Experimentos (DoE) e Controle Estatístico de Processo (CEP) . Já as ferramentas são mais bem definidas e de aplicação mais imediata. Uma ferramenta geralmente é estreita no foco e costumeiramente é usada por si só (sem a necessidade de outras ferramentas). São exemplos de ferramentas: diagrama de relações, diagrama de causa efeito, gráfico de controle e histograma. Pode-se acrescentar que uma técnica específica 
também pode ser usada para suportar diferentes princípios e uma mesma ferramenta pode ser útil dentro de várias técnicas.

Segundo Dale e McQuarter (1998) as técnicas e ferramentas da qualidade mais comumente utilizados pelas empresas, estão apresentados no Quadro 2. Vale destacar que alguns deles, como benchmarking, brainstorming e fluxograma, são instrumentos utilizados também em diversas outras áreas da gestão além da qualidade.

Quadro 2 - Técnicas e ferramentas da qualidade mais utilizadas

\begin{tabular}{|c|c|c|}
\hline $\begin{array}{c}\text { As 7 ferramentas estatísticas da qualidade e as } \\
\text { ferramentas gerencias da qualidade }\end{array}$ & Outras ferramentas & Técnicas \\
\hline Diagrama de causa e efeito & Brainstorming & Benchmarking \\
Folha de verificação & $5 \mathrm{~W} 2 \mathrm{H}$ & Desenho de experimentos \\
Gráfico de controle & Fluxograma & FMEA \\
Estratificação & Amostragem & Poka yoke \\
Histograma & & MASP \\
Gráfico de Pareto & & Custo da qualidade \\
Diagrama de dispersão & QFD \\
Diagrama de relações & & \\
Diagrama de afinidades & & \\
Diagrama de árvore & & \\
Matriz de priorização & & \\
Matriz de relações & & \\
Diagrama de processo decisório & & \\
Diagrama de setas & & \\
\hline
\end{tabular}

Fonte: Dale e McQuarter (1998)

As T\&Fs utilizadas neste estudo foram selecionadas a partir dos trabalhos de Dale e McQuarter (1998), Satolo et al. (2009) e Oliveira et al. (2011).

\section{Metodologia}

Este trabalho tem natureza quantitativa com caráter descritivo e exploratório (BRYMAN, 2012). O procedimento de coleta de dados utilizado foi o método survey, caracterizado por definição de amostra e aplicação de questionários (BABBIE, 2007).

$\mathrm{O}$ teste-piloto do questionário possibilitou identificar pontos de melhoria do instrumento, tal como a melhoria da redação e fusão de algumas questões semelhantes. $\mathrm{O}$ questionário final foi desenvolvido através de uma ferramenta do Google Docs e enviado via email para os responsáveis da direção dos programas de qualidade das empresas constantes da amostra pretendida.

O questionário foi preparado seguindo as recomendações da literatura científica. Ele foi estruturado em três seções principais: caracterização da empresa, questões relacionadas aos programas, técnicas e ferramentas da qualidade e questões relativas à acreditação.

As questões do questionário, excetuando aquelas referentes à primeira seção (caracterização da empresa), continham uma afirmativa com 5 alternativas baseadas na escala Likert.

O bloco de questões relacionadas às T\&Fs da qualidade verificar o grau de utilização nas respectivas organizações, que eram: a) não conhece, b) não pretende implementar, c) pretende implementar no futuro, d) está sendo implementado no momento e e) já foi implementado.

Na seção a respeito da acreditação, para verificar o grau de concordância dos respondentes em relação os benefícios e dificuldades, foi utilizado a escala a seguir: a) discordo completamente; b) discordo parcialmente; c) não concordo e nem discordo; d) concordo parcialmente; e e) concordo completamente. Para permitir a tabulação e análise dos dados, a estas alternativas foram associados escores que variaram de 1,0 a 5,0, sendo 1,0 para a alternativa discordo completamente e 5,0 para concordo completamente. Logo, respostas com 
valores situados entre 1,0 e 3,0 discordam da afirmativa posta, respostas com valor 3,0 não concordam nem discordam da afirmativa e valores acima de 3,0 concordam com a afirmativa.

As respostas foram tabuladas, tratadas e analisadas com o auxílio do software SPSS ${ }^{\circledR}$ (Statistical Package for the Social Sciences). Foi realizada uma análise em relação às médias de cada uma das questões e depois a correlação estatística entre as mesmas.

O questionário foi enviado às organizações acreditadas, conforme a Organização Nacional de Acreditação (ONA, 2014), que possuíam certificações válidas em março de 2014. O conjunto de empresas acreditadas utilizadas neste trabalho é composto por 399 das possíveis organizações prestadoras de serviços de saúde. A aplicação foi realizada entre os meses de agosto de 2014 a janeiro 2015.

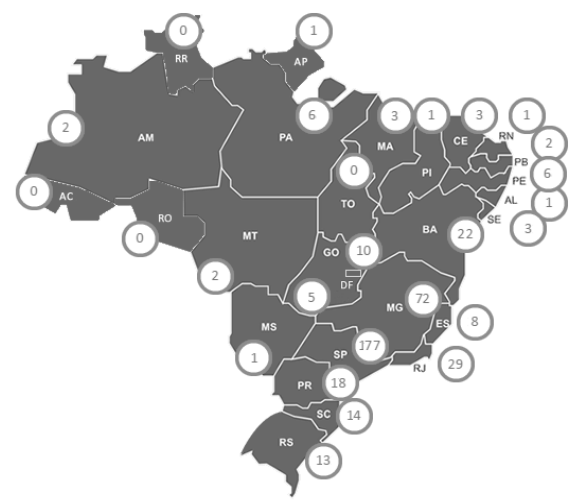

Figura 1 - Empresas com certificação válidas utilizadas para pesquisa Fonte: ONA (2014)

A Figura 1 apresenta a distribuição de certificações válidas por estados no território brasileiro. A região Sudeste concentra 71,7\% (286) das certificações, seguida pela região Sul, $11,3 \%$ (45), região Nordeste, $10,3 \%$ (41), região Centro-Oeste, $4,5 \%$ (18), e por fim, região Norte com apenas 2,3\% (9) de certificações válidas.

\section{Análise e discussão dos resultados}

Esta seção do texto caracteriza o grupo de empresas participantes da pesquisa e apresenta e analisa os principais dados obtidos com a aplicação do questionário.

O Quadro 3 apresenta o quantitativo de empresas participantes da pesquisa.

Quadro 3 - Empresas participantes da pesquisa

\begin{tabular}{|c|c|c|}
\hline Tipo da empresa & Quantidade & Frequência (\%) \\
\hline Hospital & 54 & $64,3 \%$ \\
\hline Laboratório & 13 & $15,5 \%$ \\
\hline Ambulatório & 9 & $10,7 \%$ \\
\hline Hemoterapia & 3 & $3,6 \%$ \\
\hline Diagnóstico por imagem, radioterapia e medicina nuclear & 3 & $3,6 \%$ \\
\hline Nefrologia e terapia renal substitutiva & 1 & $1,2 \%$ \\
\hline Programas da saúde e prevenção de riscos & 1 & $1,2 \%$ \\
\hline
\end{tabular}

Fonte: Elaborado pelo autor (2015)

Pelo exposto na Quadro 3, os hospitais apresentam o maior número de empresas estudadas, seguidos pelos laboratórios, ambulatórios, organizações de diagnóstico por imagem, radioterapia e medicina nuclear, hemoterapia, nefrologia e terapia renal substitutiva e programas de saúde e prevenção de riscos. Cabe destacar que a pesquisa buscou manter a proporção de empresas selecionadas na amostra tal como os estratos da população.

O Quadro 4 apresenta o porte das empresas prestadoras de serviços respondentes adotados pelo Serviço Brasileiro de Apoio às Micro e Pequenas Empresas (SEBRAE). A 
maioria das empresas participantes da pesquisa é de grande porte e nesse sentido, deve ficar claro que esta é uma limitação do estudo, pois as conclusões estão restritas ao porte das empresas (principalmente grandes). Não houve neste estudo organizações respondentes classificadas como microempresa (até 19 empregados).

Quadro 4 - Porte das empresas participantes da pesquisa

\begin{tabular}{|c|c|c|}
\hline Porte da empresa & Quantidade & Frequência (\%) \\
\hline de 10 a 49 empregados (pequena) & 13 & $15,5 \%$ \\
\hline de 50 a 99 empregados (média) & 7 & $8,3 \%$ \\
\hline mais de 100 empregados (grande) & 64 & $76,2 \%$ \\
\hline
\end{tabular}

Fonte: Elaborado pelo autor (2015)

A Figura 2 apresenta a duração dos processos de obtenção da primeira certificação. A maioria das empresas estudadas levou de 7 a 12 meses para obter a certificação. Tal fato pode ser explicado tendo em vista que grande parte das organizações teve o auxílio de consultorias especializadas, conforme mostrado na Figura 3. Tais empresas de consultoria possuem procedimentos padrões preestabelecidos rígidos para implantação de certificações e, por esse motivo, os prazos para o seu desenvolvimento e implantação ficam muito próximos independentemente do tipo de empresa.

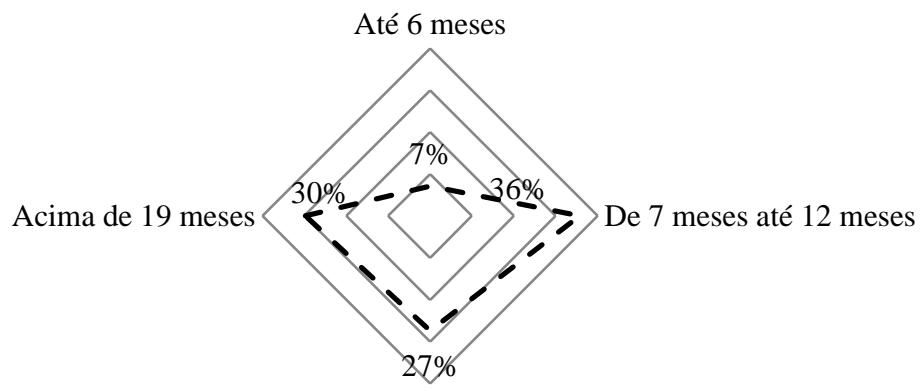

De 13 meses até 18 meses

Figura 2 - Tempo para obtenção da primeira certificação Fonte: Elaborado pelo autor (2015)

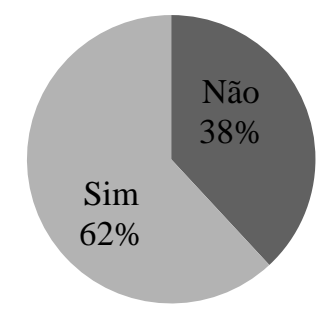

Figura 3 - Utilização de consultoria para a Acreditação

Fonte: Elaborado pelo autor (2015)

Verifica-se que a maioria delas utilizou consultoria externa para obter a certificação. Apesar das empresas de consultoria apresentarem excessiva padronização do processo e dos instrumentos de implantação de certificações, seu suporte e expertise são interessantes e importantes elementos para viabilizar, otimizar e reduzir o tempo de desenvolvimento e implantação desse tipo de sistema nas empresas em geral. 
A Quadro 5 apresenta a distribuição das empresas estudadas pelo tipo de certificado. As frequências encontradas seguem a mesma distribuição do universo utilizado na pesquisa, razoavelmente homogênea.

Quadro 5 - Tipos de certificações das empresas estudadas

\begin{tabular}{|c|c|c|}
\hline Tipo de Certificação & Quantidade & Frequência \\
\hline Acreditado & 21 & $25 \%$ \\
\hline Acreditado Pleno & 30 & $36 \%$ \\
\hline Acreditado com Excelência & 33 & $39 \%$ \\
\hline
\end{tabular}

Fonte: Elaborado pelo autor (2015)

Em média a empresas necessitam 2,4 anos para a transição da certificação Acreditado para Acreditado Pleno, 2,5 anos de Acredito Pleno para Acreditado com Excelência e 3,9 anos de Acreditado para Acreditado com Excelência. Esses resultados podem ser explicados tendo vista que o prazo de validade das certificações Acreditado e Acreditado Pleno é de dois anos e o Acreditado com Excelência tem validade de 3 anos.

Quase todas as empresas estudadas possuem um setor de qualidade como forma de dar suporte ao desenvolvimento da qualidade. Isso significa um efetivo e interessante investimento na melhoria contínua a partir de recursos humanos próprios (Ver Figura 4).

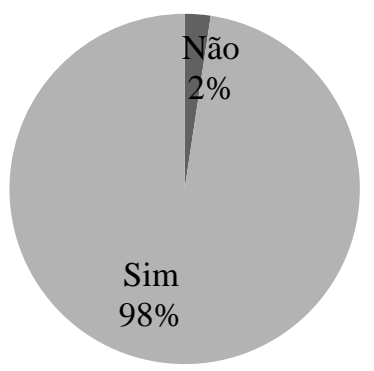

Figura 4 - Tempo para obtenção da primeira certificação

Fonte: Elaborado pelo autor (2015)

Quanto ao tempo de existência de um departamento de qualidade institucional, observou-se que 39,3\% das organizações possuíam tal órgão entre 6 e 10 anos, 35,7\% entre 2 e 5 anos, 20,2\% a mais de anos, 3,6\% das organizações não possuíam departamento de qualidade e 1,2\% há um ano ou menos (Ver Quadro 6).

Quadro 6 - Tempo de existência do departamento de qualidade

\begin{tabular}{|c|c|c|}
\hline Há quanto tempo possui um departamento de qualidade? & Quantidade & Frequência \\
\hline Há um ano ou menos & 1 & $1,2 \%$ \\
\hline Entre 2 e 5 anos & 30 & $35,7 \%$ \\
\hline Entre 6 e 10 anos & 33 & $39,3 \%$ \\
\hline Mais de 10 anos & 17 & $20,2 \%$ \\
\hline Não possui departamento & 3 & $3,6 \%$ \\
\hline
\end{tabular}

Fonte: Elaborado pelo autor (2015)

Foi questionado aos gestores "Quando a gestão da qualidade passou a ser uma preocupação?" A maioria dos respondentes afirmou que esta preocupação surgiu nos últimos dez anos (33,3\%), seguindo por nos últimos cinco anos ou $(26,2 \%)$, desde a fundação $(25 \%)$ e nos últimos 20 anos (15,5\%) (Ver Quadro 7). Tais resultados podem ser explicados pela fundação da ONA em 1999. Além disso, a questão da garantia da qualidade é a tônica das discussões dos rumos da medicina nos últimos anos. 
Quadro 7 - Preocupação da instituição com a Gestão da Qualidade

\begin{tabular}{|c|c|c|}
\hline Quando a Gestão da Qualidade passou a ser uma preocupação da instituição? & Quantidade & Frequência \\
\hline Nos últimos 10 anos & 28 & $33,3 \%$ \\
\hline Nos últimos 5 anos & 22 & $26,2 \%$ \\
\hline Desde sua fundação & 21 & $25 \%$ \\
\hline Nos últimos 20 anos & 13 & $15,5 \%$ \\
\hline
\end{tabular}

Fonte: Elaborado pelo autor (2015)

A pesquisa verificou quais os principais ferramentas da qualidade são utilizados pelas empresas estudadas (Ver Quadro 8).

Quadro 8 - Ferramentas da qualidade implantadas e não implantadas nas organizações estudadas

\begin{tabular}{|c|c|c|c|c|c|}
\hline Ferramentas & Não conhece & $\begin{array}{c}\text { Não } \\
\text { pretende } \\
\text { implementar }\end{array}$ & $\begin{array}{l}\text { Pretende implementar } \\
\text { no futuro }\end{array}$ & $\begin{array}{c}\text { Está sendo } \\
\text { implementado } \\
\text { no momento }\end{array}$ & $\begin{array}{c}\text { Já foi } \\
\text { implementado }\end{array}$ \\
\hline Fluxograma & $0,00 \%$ & $2,40 \%$ & $0,00 \%$ & $2,40 \%$ & $95,20 \%$ \\
\hline $\begin{array}{l}\text { Procedimento operacional } \\
\text { padrão } \\
\end{array}$ & $0,00 \%$ & $2,40 \%$ & $0,00 \%$ & $2,40 \%$ & $95,20 \%$ \\
\hline $5 \mathrm{~W} 2 \mathrm{H}$ & $4,80 \%$ & $1,20 \%$ & $1,20 \%$ & $3,60 \%$ & $89,30 \%$ \\
\hline Diagrama de causa e efeito & $2,40 \%$ & $1,20 \%$ & $6,00 \%$ & $3,60 \%$ & $86,90 \%$ \\
\hline Brainstorming & $3,60 \%$ & $3,60 \%$ & $4,80 \%$ & $3,60 \%$ & $84,50 \%$ \\
\hline Folha de verificação & $7,10 \%$ & $11,90 \%$ & $4,80 \%$ & $6,00 \%$ & $70,20 \%$ \\
\hline Gráfico de controle & $8,30 \%$ & $10,70 \%$ & $6,00 \%$ & $6,00 \%$ & $69,00 \%$ \\
\hline Gráfico de Pareto & $0,00 \%$ & $14,30 \%$ & $11,90 \%$ & $6,00 \%$ & $67,90 \%$ \\
\hline Estratificação & $10,70 \%$ & $16,70 \%$ & $10,70 \%$ & $3,60 \%$ & $58,30 \%$ \\
\hline Diagrama de priorização & $19,00 \%$ & $10,70 \%$ & $19,00 \%$ & $3,60 \%$ & $47,60 \%$ \\
\hline Histograma & $6,00 \%$ & $31,00 \%$ & $16,70 \%$ & $2,40 \%$ & $44,00 \%$ \\
\hline Diagrama de dispersão & $13,10 \%$ & $34,50 \%$ & $26,20 \%$ & $3,60 \%$ & $22,60 \%$ \\
\hline Diagrama de matriz & $26,20 \%$ & $40,50 \%$ & $9,50 \%$ & $1,20 \%$ & $22,60 \%$ \\
\hline $\begin{array}{c}\text { Diagrama de processo } \\
\text { decisório }\end{array}$ & $26,20 \%$ & $26,20 \%$ & $17,90 \%$ & $7,10 \%$ & $22,60 \%$ \\
\hline Diagrama de setas & $28,60 \%$ & $36,90 \%$ & $9,50 \%$ & $3,60 \%$ & $21,40 \%$ \\
\hline Diagrama de relações & $31,00 \%$ & $36,90 \%$ & $10,70 \%$ & $1,20 \%$ & $20,20 \%$ \\
\hline Diagrama de árvore & $32,10 \%$ & $39,30 \%$ & $13,10 \%$ & $1,20 \%$ & $14,30 \%$ \\
\hline Diagrama de afinidade & $34,50 \%$ & $41,70 \%$ & $13,10 \%$ & $2,40 \%$ & $8,30 \%$ \\
\hline ServQual & $58,30 \%$ & $23,80 \%$ & $8,30 \%$ & $1,20 \%$ & $8,30 \%$ \\
\hline
\end{tabular}

Fonte: Elaborado pelo autor (2015)

É possível classificar as ferramentas da qualidade segundo as ferramentas já implementadas pelas empresas participantes em três grupos: os mais utilizados (procedimento operacional padrão, fluxograma, 5W2H, diagrama de causa e efeito e brainstorming), os medianamente utilizados (folha de verificação, gráfico de controle, gráfico de Pareto, estratificação, diagrama de priorização, histograma, diagrama de processo decisório, diagrama 
de matriz, diagrama de dispersão e diagrama de setas e diagrama de relações) e os menos utilizados (diagrama de árvore, ServQual e diagrama de afinidade).

Os instrumentos mais utilizados são os mais simples e considerados "alicerces" para a evolução do SGQ e para a adoção de técnicas e ferramentas mais complexa. Ressalta-se que o digrama de causa e efeito é o único elemento das 7 ferramentas da qualidade básicas utilizadas pelas organizações com maior intensidade.

Alguns dos instrumentos classificados no grupo dos medianamente utilizados causam certa estranheza, dado seu baixo grau de complexidade. Assim tais ferramentas poderiam estar tranquilamente no grupo dos mais utilizados. São eles: folha de verificação, gráfico de controle, gráfico de Pareto, histograma e estratificação. Acredita-se que os respondentes utilizem alguma forma adaptada deles ou mesmo não conheçam a nomenclatura utilizada neste estudo.

O terceiro grupo é nitidamente composto por elementos com maior grau de complexidade e que exige maior maturidade em termos de gestão das organizações para os utilizarem, apesar do alto retorno em termos de resultado que podem gerar. Ressalta-se aqui certa preocupação pela não utilização do ServQual tendo em vista que esta é ferramenta mais popular para realizar medições de qualidade em serviços pela percepção do consumidor final em empresas prestadoras de serviços.

As técnicas mais utilizadas foram ciclo PDCA, Controle Estatístico de Processo, FMEA e 5S, mas em contrapartida, QFD e DoE foram as técnicas menos implantadas (Ver Quadro 9).

Quadro 9 - Técnicas da qualidade implantadas e não implantadas nas organizações estudadas

\begin{tabular}{|c|c|c|c|c|c|}
\hline Técnicas & Não conhece & $\begin{array}{c}\text { Não pretende } \\
\text { implementar }\end{array}$ & $\begin{array}{c}\text { Pretende implementar } \\
\text { no futuro }\end{array}$ & $\begin{array}{c}\text { Está sendo } \\
\text { implementado no } \\
\text { momento }\end{array}$ & $\begin{array}{c}\text { Já foi } \\
\text { implementado }\end{array}$ \\
\hline Ciclo PDCA & $0,0 \%$ & $1,2 \%$ & $1,2 \%$ & $4,8 \%$ & $92,9 \%$ \\
\hline $\begin{array}{c}\text { FMEA - Análise dos } \\
\text { Modos de Falha e } \\
\text { Efeitos }\end{array}$ & $2,4 \%$ & $9,5 \%$ & $15,5 \%$ & $10,7 \%$ & $61,9 \%$ \\
\hline $\begin{array}{c}\text { Controle estatístico } \\
\text { de processo }\end{array}$ & $4,8 \%$ & $9,5 \%$ & $13,1 \%$ & $10,7 \%$ & $61,9 \%$ \\
\hline $5 S$ & $1,2 \%$ & $11,9 \%$ & $16,7 \%$ & $9,5 \%$ & $60,7 \%$ \\
\hline $\begin{array}{c}\text { QFD - } \\
\text { Desdobramento da } \\
\text { Função Qualidade }\end{array}$ & $32,1 \%$ & $25,0 \%$ & $10,7 \%$ & $6,0 \%$ & $26,2 \%$ \\
\hline $\begin{array}{c}\text { DoE - Delineamento } \\
\text { de experimentos }\end{array}$ & $56,0 \%$ & $29,8 \%$ & $8,3 \%$ & $0,0 \%$ & $6,0 \%$ \\
\hline
\end{tabular}

Fonte: Elaborado pelo autor (2015)

Em geral, não houve surpresa em relação às técnicas utilizadas, tendo em vista o nível de maturidade da Gestão da Qualidade, bem como, o período de tempo no qual a qualidade se tornou uma preocupação por parte das organizações. Assim, é esperado que técnicas mais simples (ciclo PDCA) estejam mais difundidas do que as mais complexas (DoE - Delineamento de experimentos).

A pesquisa verificou a existência de diferenças significativas no uso de T\&Fs dado o tipo de certificação das organizações. As T\&Fs, por serem variáveis categóricas codificadas com valores de 1 a 5 para este estudo, foram transformar em variável dicotômica (1 implantadas e 2 - não implementado) para as análises.

Em geral, utilizado o teste Qui-quadrado, podemos dizer que não há diferenças significativas no uso de T\&Fs por tipo de certificações, ou seja, não há dependência entre se a T\&F é implementada ou não e tipo de certificação, exceto em cinco itens, como: estratificação, gráfico de Pareto, histograma, diagrama de dispersão, e diagrama de priorização (Ver Quadro $10)$. 
Quadro 10 - Testes estatísticos entre tipos de Acreditação

\begin{tabular}{|c|c|c|c|c|c|}
\hline \multirow{2}{*}{ Técnicas e Ferramentas da qualidade } & \multicolumn{2}{|c|}{$\%$ de empresas que possuem técnicas e ferramentas da } & \multicolumn{2}{c|}{ Teste estatístico } \\
\cline { 2 - 6 } & \multirow{2}{*}{ Acreditado } & $\begin{array}{c}\text { Acreditade implantadas } \\
\text { Pleno }\end{array}$ & Acreditado com Excelência & Qui-quadrado & Sig. \\
\hline Estratificação & $18,4 \%$ & $28,6 \%$ & $53,1 \%$ & 9,430 & $0,009^{*}$ \\
\hline Folha de Verificação & $22,0 \%$ & $35,6 \%$ & $42,4 \%$ & 1,179 & 0,555 \\
\hline Gráfico de Pareto & $15,8 \%$ & $35,1 \%$ & $49,1 \%$ & 10,405 & $0,006^{*}$ \\
\hline Diagrama de Causa e Efeito & $24,7 \%$ & $32,9 \%$ & $42,5 \%$ & 2,718 & 0,257 \\
\hline Histograma & $18,9 \%$ & $27,0 \%$ & $54,1 \%$ & 6,047 & $0,049^{*}$ \\
\hline Diagrama de dispersão & $15,8 \%$ & $10,5 \%$ & $73,7 \%$ & 12,590 & $0,002^{*}$ \\
\hline Gráfico de controle & $25,9 \%$ & $31,0 \%$ & $43,1 \%$ & 1,900 & 0,387 \\
\hline Diagrama de afinidade & $0,0 \%$ & $28,6 \%$ & $71,4 \%$ & 4,026 & 0,134 \\
\hline Diagrama de relações & $11,8 \%$ & $35,3 \%$ & $52,9 \%$ & 2,506 & 0,286 \\
\hline Diagrama de árvore & $0,0 \%$ & $50,0 \%$ & $50,0 \%$ & 4,709 & 0,095 \\
\hline Diagrama de matriz & $15,8 \%$ & $36,8 \%$ & $47,4 \%$ & 1,250 & 0,535 \\
\hline Diagrama de priorização & $22,5 \%$ & $25,0 \%$ & $52,5 \%$ & 6,040 & $0,049^{*}$ \\
\hline Diagrama de processo decisório & $5,3 \%$ & $47,4 \%$ & $47,4 \%$ & 5,168 & 0,075 \\
\hline Diagrama de setas & $16,7 \%$ & $33,3 \%$ & $50,0 \%$ & 1,342 & 0,511 \\
\hline Fluxograma & $23,8 \%$ & $36,3 \%$ & $40,0 \%$ & 1,403 & 0,496 \\
\hline 5W2H & $24,0 \%$ & $36,0 \%$ & $40,0 \%$ & 0,387 & 0,824 \\
\hline Brainstorming & $26,8 \%$ & $32,4 \%$ & $40,8 \%$ & 2,269 & 0,322 \\
\hline ServQual & $14,3 \%$ & $57,1 \%$ & $28,6 \%$ & 1,556 & 0,459 \\
\hline Operacional Padrão & $25,0 \%$ & $35,0 \%$ & $40,0 \%$ & 0,458 & 0,795 \\
\hline PDCA & $24,4 \%$ & $35,9 \%$ & $39,7 \%$ & 0,248 & 0,883 \\
\hline QFD & $18,2 \%$ & $40,9 \%$ & $40,9 \%$ & 0,799 & 0,670 \\
\hline FMEA & $17,3 \%$ & $36,5 \%$ & $46,2 \%$ & 4,896 & 0,086 \\
\hline PS & $27,5 \%$ & $33,3 \%$ & $39,2 \%$ & 0,518 & 0,772 \\
\hline DoE & $20,0 \%$ & $20,0 \%$ & $60,0 \%$ & 1,001 & 0,606 \\
\hline CEP & $21,2 \%$ & $36,5 \%$ & $42,3 \%$ & 1,151 & 0,562 \\
\hline
\end{tabular}

Fonte: Elaborado pelo autor (2015)

Buscou-se, nesta pesquisa, identificar quais os principais benefícios, de uma forma geral, auferidos com a implantação da Acreditação (Ver Figura 5).

A "Melhoria no controle dos processos da organização", "Aumento na Segurança do paciente", e "Melhoria no gerenciamento de recursos" obtiveram os maiores escores, 4,87, 4,81, e 4,71, respectivamente. Esse fato corrobora os principais objetivos da Acreditação, orientação por processos e resultados, bem como o foco na segurança do paciente. O bom desempenho nesses itens aumenta consideravelmente as chances de distinção da organização, aos clientes internos e externos.

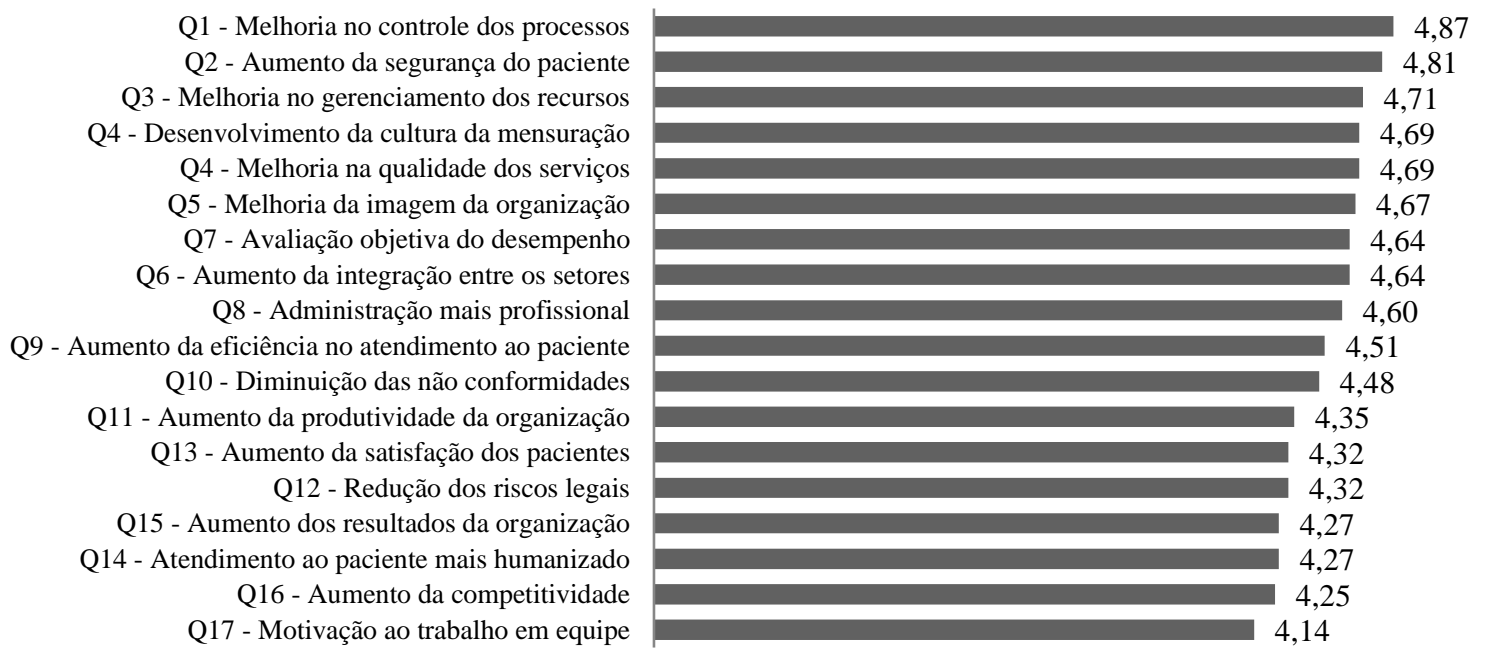

Figura 5 - Benefícios auferidos com a Acreditação

Fonte: Elaborado pelo autor (2015)

As próximas cinco questões (Q4, Q5, Q6, Q7, Q8 e Q9) apresentaram escores também significativos (média acima de 4,5), confirmando que a Acreditação também proporciona 
melhoria na qualidade dos serviços, melhoria na imagem da organização, avaliação objetiva do desempenho, aumento da integração dos setores, gestão mais profissional e aumento na eficiência no atendimento do paciente.

Todos os demais benefícios provenientes da Acreditação contidos nas questões de Q10 a Q17 também foram confirmados, porém com menor intensidade que os anteriores. São eles: diminuição das não conformidades, aumento da produtividade, aumento da satisfação do cliente, redução dos riscos legais, aumento dos resultados da organização, atendimento mais humanizado, aumento da competitividade e motivação ao trabalho em equipe.

As principais dificuldades enfrentadas pelas organizações pesquisadas para obter a Acreditação são apresentadas na Figura 6.

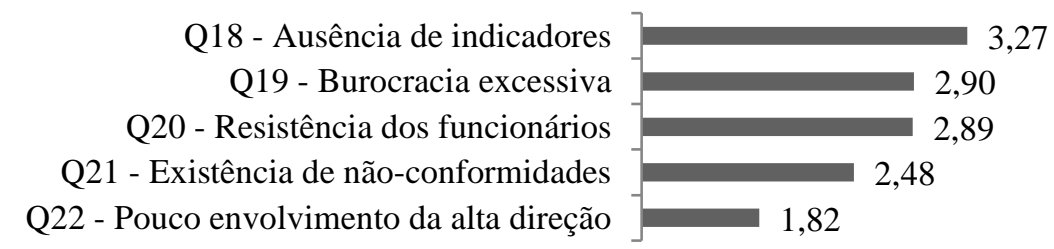

Figura 6 - Dificuldades enfrentadas na implantação da Acreditação

Fonte: Elaborado pelo autor (2015)

A questão Q18 que afirmava que a ausência de indicadores era uma dificuldade para implantação do sistema, apresentou um escore acima de 3,0, caracterizando que os respondentes concordam razoavelmente.

Pode-se observar que a burocracia excessiva e resistência dos funcionários relativos às questões Q19 e Q20, foram classificadas pelos respondentes com um escore muito próximo de 3,0 , o que significa que não são dificuldades nem benefícios do ponto de vista deles.

Já as questões 21 e 22, que afirmava que a existência de não conformidades e o baixo envolvimento da alta direção era uma dificuldade para implantação do sistema, apresentou um escore abaixo de 3,0, caracterizando que os respondentes discordam consideravelmente delas.

\section{Considerações finais}

A Acreditação e as técnicas e ferramentas da qualidade representam diferenciais importantes para as organizações, pois proporcionam benefícios de diversas ordens (tanto internos como externos) conforme os dados apresentados neste trabalho. Nesse sentido, a pesquisa ratificou o que já era relatado na teoria científica, evidenciando que as bases deste sistema são aplicáveis à realidade do setor de saúde.

O objetivo deste trabalho foi verificar as principais características do processo de Acreditação, seus benefícios, suas dificuldades e quais técnicas e ferramentas da qualidade eram utilizados em empresas brasileiras.

As ferramentas da qualidade mais utilizadas foram: procedimento operacional padrão, fluxograma, $5 \mathrm{~W} 2 \mathrm{H}$, diagrama de causa e efeito e brainstorming. Constatou-se que várias ferramentas básicas da qualidade não estão sendo utilizadas. Ressalta-se, também, certa preocupação pela não utilização do ServQual, ferramenta exclusiva para avaliação da qualidade na área de serviços. Quanto às técnicas, aquelas receberam maior destaque foram: ciclo PDCA, FMEA e CEP. Tais técnicas apresentam grau de complexidade baixo a médio e, portanto, já era esperado encontra-las como as mais difundidas. 
Pode-se citar, pelos resultados obtidos nesta pesquisa, que a Acreditação gera benefícios significativos às organizações, tais como: melhoria no controle dos processos da organização, aumento na segurança do paciente e melhoria no gerenciamento de recursos.

As dificuldades de desenvolvimento e implantação desses sistemas se confirmaram para a amostra pesquisada. Apenas a ausência de indicadores ficou com escore 3,27, podendo ser considerado que os respondentes concordam levemente. Esse fato pode estar relacionado com a falta de cultura de utilização de indicadores para controle gerencial nas organizações estudadas. As demais dificuldades não foram consideradas, e tal fato pode estar relacionado com o alto índice de utilização de consultorias especializadas para implantação da Acreditação.

Por fim, ressalta-se que esta pesquisa apresenta um quadro geral sobre o tema e gerou informações que podem servir de base para outros trabalhos complementares e/ou mais específicos: em outras regiões, em tipos específicos de organizações (hospitais, laboratórios, entre outros) ou ainda complementando e aprofundando os resultados aqui obtidos.

\section{Referências bibliográficas}

ANTUNES, L. M. Implantação do processo de acreditação baseado no manual das organizações prestadoras de serviços hospitalares da ONA. 2002. 102 f. Dissertação (Mestrado em Engenharia) - Universidade do Rio Grande do Sul, Porto Alegre, 2002.

BABBIE, E. Métodos de pesquisas de survey. Belo Horizonte: Editora UFMG, 2007.

BAMFORD, D. R.; GREATBANKS, R. W. The use of quality management tools and techniques: a study of application in everyday situations. International Journal of Quality \& Reliability Management, v. 22, n. 4, p. 376-392, 2005.

BRYMAN, A. Social research methods. Oxford: Oxford University Press, 2012.

CARPINETTI, L. C. R. Gestão da qualidade: conceitos e técnicas. São Paulo: Atlas, 2012.

CARPINETTI, L. C. R.; GEROLAMO, M. C.; MIGUEL, P. A. C. Gestão da Qualidade ISO 9001:2008: princípios e requisitos. São Paulo: Atlas, 2011.

CARVALHO, M. M. DE; PALADINI, E. P. Gestão da Qualidade: teoria e casos. Rio de Janeiro: Elsevier Campus, 2012.

CORREA, H. L.; CAON, M. Gestão de serviços: lucratividade por meio de operações e de satisfação dos clientes. São Paulo: Atlas, 2009.

COUTO, R. C.; PEDROSA, T. M. G. Hospital: acreditação e gestão em saúde. Rio de Janeiro: Guanabara Koogan, 2007.

COUTO, R. C.; PEDROSA, T. M. G. Hospital: gestão operacional e sistemas de garantia de qualidade viabilizando a sobrevivência. Rio de Janeiro: Medsi, 2003.

DALE, B. G.; MCQUATER, R. Managing business improvement and quality: implementing key tools and techniques. Oxford, UK ; Malden, Mass: Blackwell Business, 1998.

FITZSIMMONS, J. A.; FITZSIMMONS, M. J. Administração de Serviços: operações, estratégias e tecnologia da informação. Porto Alegre: Bookman, 2014.

HELLSTEN, U. \& KLEFSJÖ, B. TQM as a management system consisting of values, techniques and tools. The TQM Magazine. v. 12, n. 4, pp. 238-244, 2000.

HELLSTEN, U.; KLEFSJÖ, B. TQM as a management system consisting of values, techniques and tools. The TQM Magazine, v. 12, n. 4, p. 238-244, 2000. 
KOHN, L. T.; CORRIGAN, J.; DONALDSON, M. S. To err is human: building a safer health system. Washington, D.C: National Academy Press, 2000.

MALIK, A. M.; SCHIESARI, L. C. M. Qualidade na gestão local de serviços e ações de saúde. São Paulo: Fundação Petrópolis, 1998.

McQUATER R.E., DALE B.G., BOADEN R.J., WILCOX M. The effectiveness of quality management tools and techniques: an examination of the key influences in five plants, In: Proceedings of the Institution of Mechanical Engineers, Part. B: Journal of Engineering Manufacture, 210, 329-339, 1996.

MCQUATER, R. E.; SCURR, C. H.; DAL, B. G.; HILLMAN, P. G. Using quality tools and techniques successfully. The TQM Magazine, v. 7, n. 6. pp.37-42, 1995.

OLIVEIRA, J. A.; NADAE, J.; OLIVEIRA, O. J., SALGADO, M. H. Um estudo sobre a utilização de sistemas, programas e ferramentas da qualidade em empresas do interior de São Paulo. Produção, São Paulo, v. 21, n. 4, p. 708-723, 2011.

ORGANZAÇÃO NACIONAL DE ACREDITAÇÃO (ONA). Disponível em: <www.ona.org.br>. Acessos em: 15 out. 2015.

ORGANZAÇÃO NACIONAL DE ACREDITAÇÃO (ONA). Manual das Organizações Prestadoras de Serviços de Saúde. Brasília: ONA, 2010.

PAGANINI, J. M.; NOVAES, H. DE M. Garantia de qualidade: acreditaçäo de hospitais para América Latina e o Caribe. OPAS. Série Silos, v. 13, 1992.

PEDREIRA, M. L. G. Errar é humano: estratégias para a busca da segurança do paciente. In: Harada, et al. O erro humano e a segurança do paciente. São Paulo: Atheneu, 2006.

PEDROSO, M. C.; MALIK, A. M. As quatro dimensões competitivas da saúde. Harvard Business Review, v. 89, n. 3, p. 54-63, 2011.

QUINTO NETO, A.; GASTAL, F. L. Acreditação hospitalar: proteção aos usuários dos profissionais e das instituições de saúde. Porto Alegre: Dacasa, 2000.

RODRIGUES, M. V; CARÂP, L. J.; EL-WARRACK, L. O.; REZENDE, T. B. Qualidade e acreditação em saúde. São Paulo: FGV Editora, 2011.

ROONEY, A. L.; VAN OSTENBERG, P. R. Licenciamento, acreditação e certificação: abordagens à qualidade de serviços de saúde. Bethesda: Center for Human Services, 1999. (Série de Aperfeiçoamento Sobre a Metodologia de Garantia de Qualidade).

SATOlO, E. G.; ANDRIETTA, J. M.; MIGUEL, P. A. C.; CALARGE, F. A. Análise da utilização de técnicas e ferramentas no programa Seis Sigma a partir de um levantamento tipo survey. Produção, São Paulo, v. 19, n. 2, p. 400-416, 2009.

ZEITHAML, V. A.; FARRELL, M. J.; GREMLER, D. D. Marketing de serviços: a empresa com foco no cliente. Porto Alegre: Bookman, 2014. 